\title{
Surface Characterization of Optics for EUV Lithography
}

\author{
PEREENED \\ D. P. Gaines, D. W. Sweeney, K. W. DeLong, \\ AlG 161906 \\ S. P. Vernon, S. L. Baker, \\ D. A. Tichenor, and R. Kestner \\ OSTI
}

This paper was prepared for submittal to the Optical Society of America Extreme Ultraviolet Lithography

Boston, MA

April 29-May 3, 1996

May 28, 1996

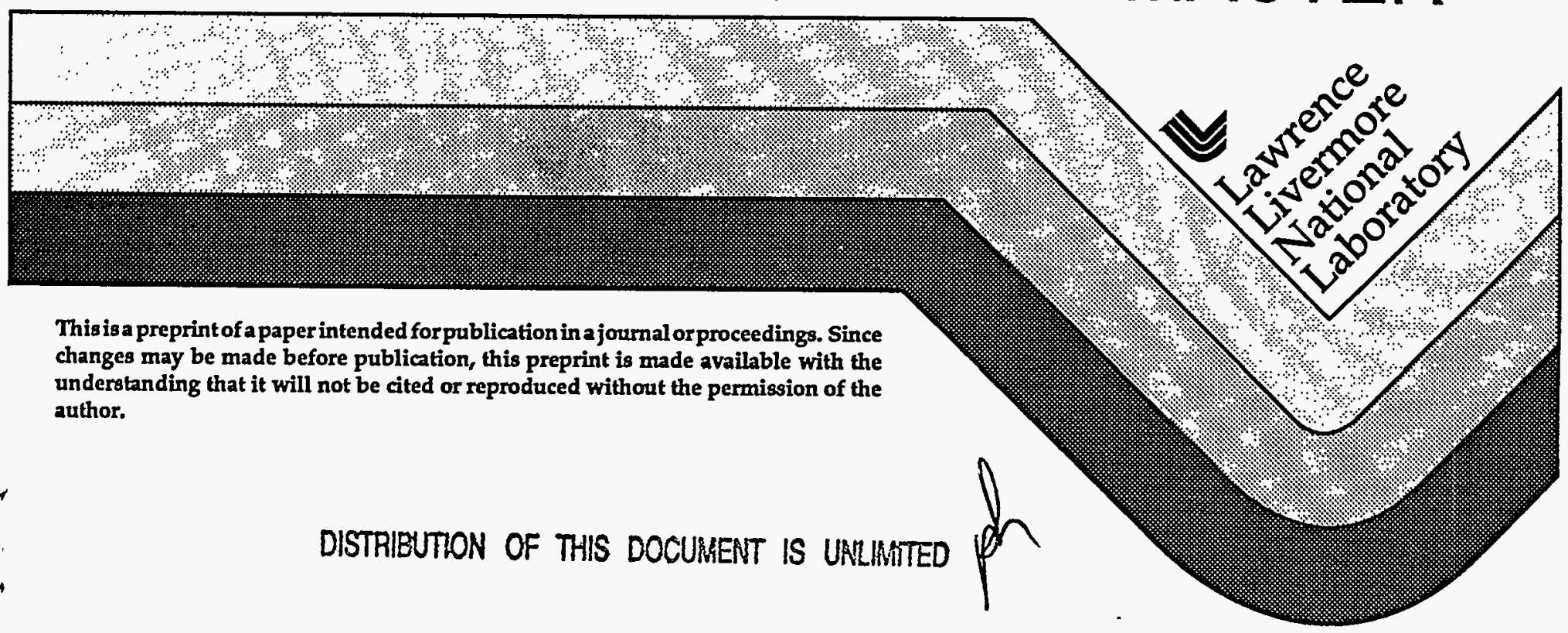




\section{DISCLAIMER}

This document was prepared as an account of work sponsored by an agency of the United States Government. Neither the United States Government nor the University of California nor any of their employees, makes any warranty, express or implied, or assumes any legal liability or responsibility for the accuracy, completeness, or usefulness of any information, apparatus, product, or process disclosed, or represents that its use would not infringe privately owned rights. Reference herein to any specific commercial product, process, or service by trade name, trademark, manufacturer, or otherwise, does not necessarily constitute or imply its endorsement, recommendation, or favoring by the United States Government or the University of California. The views and opinions of authors expressed herein do not necessarily state or reflect those of the United States Government or the University of California, and shall not be used for advertising or product endorsement purposes. 
DISCLAIMER

Portions of this document may be illegible in electronic image products. Images are produced from the best available original document. 


\title{
Surface characterization of optics for EUV lithography
}

\author{
D. P. Gaines, D.W. Sweeney, K.W. DeLong, S.P. Vernon, S.L. Baker \\ Lawrence Livermore National Laboratory, P.O. Box 808, L-395, Livermore, CA 94551 \\ D. A. Tichenor \\ Sandia National Laboratory, P.O. Box 969, Livermore, CA 94551 \\ R. Kestner \\ Tinsley Laboratories, Inc., 3900 Lakeside Drive, Richmond, CA 94806
}

\begin{abstract}
The surface topography of optics fabricated for Extreme Ultraviolet Lithography has been measured using a combination of phase-measuring interferometery and atomic force microscopy. Power Spectral Densities were computed over spatial frequencies extending from $2.0 \times 10^{-8} \mathrm{~nm}^{-1}$ to $7.7 \times 10^{-2}$ $\mathrm{nm}^{-1}$. Roughness values for frequencies greater than $1.0 \times 10^{-6} \mathrm{~nm}^{-1}$ were $0.64 \mathrm{~nm}$ rms for a spherical optic and $0.95 \mathrm{~nm}$ rms for an aspheric optic. These values are significantly larger than $0.088 \mathrm{~nm} \mathrm{rms}$, which as obtained using a spherical optic representative of current limits in surface polishing technology.
\end{abstract}

Keywords: Surface measurements-roughness, Scattering by rough surfaces, Microlithography

\section{Introduction}

The surface topography of optics for extreme ultraviolet lithography (EUVL) must be precisely controlled from spatial scales extending from the clear aperture to the wavelength of illumination.

Surface profile data obtained at low spatial frequencies can be used to deterministically predict the impulse response of the optical system, while data obtained at "mid" and higher spatial frequencies provide a statistical description of the modifications to system performance due to scattering. In this statistical regime the Power Spectral Density (PSD) representation can be related to the angular distribution of scattered radiation.
Because multilayer coatings are required in EUVL, the relationship between the PSD and scattered radiation is complicated by both the physics of thin film growth[1] and the resonant nature of the coatings.[2] In the case of growth physics, it has been shown that the surface topography of a multilayercoated substrate replicates the topography of the uncoated substrate for all spatial frequencies below a cutoff value,[3] suggesting that the interface structures are conformal at low frequencies. In the conformal limit scattering from a multilayer is equivalent to single-surface scattering; i.e., the substrate PSD can be used to describe the scattering properties of the multilayer-coated surface. Therefore, accurate characterization of the substrate surface is relevant in predicting the performance of multilayer-coated EUVL mirrors or optical systems at frequencies below the conformal limit.

Above the conformal limit the substrate PSD can be used to predict the effective PSD of the multilayer coated surface using theoretical growth models.[4]

This paper presents bare-surface PSD surface descriptions obtained for state-of-the-art EUVL optics over the entire frequency band relevant to EUVL.

\section{Experiment}

Two aspheric optics fabricated for the Sandia National Laboratory (SNL) $5 \mathrm{X}$ ring-field EUVL system[5] were characterized before coating using a Digital Instruments Dimension 5000 Atomic Force Microscope (AFM), a Zygo NewView 100 white-light interferometric microscope, a custom-built Tinsley phase-shifting interferometer, and a point-diffraction 
interferometer developed at Lawrence Livermore National Laboratory (LLNL).[6]

M1\#1 is a concave asphere $298 \mathrm{~mm}$ in diameter, with a best fit spherical radius of $1793 \mathrm{~mm}$. M2\#2 is a convex asphere with diameter $92 \mathrm{~mm}$, and best fit spherical radius of $460 \mathrm{~mm}$. Both substrates have aspheric departures of approximately $1.5 \mu \mathrm{m}$ in their respective clear apertures.

A convex spherical substrate fabricated for the LLNL EUV lithography station, and a spherical optic and an optical flat polished close to current technology limits were also characterized using the NewView 100 and AFM.

The LLNL EUVL sphere is a concave surface 75 $\mathrm{mm}$ in diameter with a radius of curvature of 137.3 $\mathrm{mm}$. The highly polished sphere is concave, $100 \mathrm{~mm}$ in diameter, and has a radius of curvature of $800 \mathrm{~mm}$. The flat is $25.4 \mathrm{~mm}$ in diameter.

Surface data was reduced to a two-dimensional PSD description in polar coordinates. Axial symmetry was assumed. Tinsley interferometric data was flattened using a procedure described elsewhere.[7] NewView data was flattened by subtracting a best-fit quadratic surface from the data. Individual scan lines of the AFM data were flattened by subtracting a bestfit first order polynomial.

\section{Results and Discussion}

PSD data for two aspheric optics fabricated for EUVL are shown in Figs. 1 and 2. Measured frequencies cover the range of frequencies relevant to EUVL. These aspheric substrates exhibit both precise figure

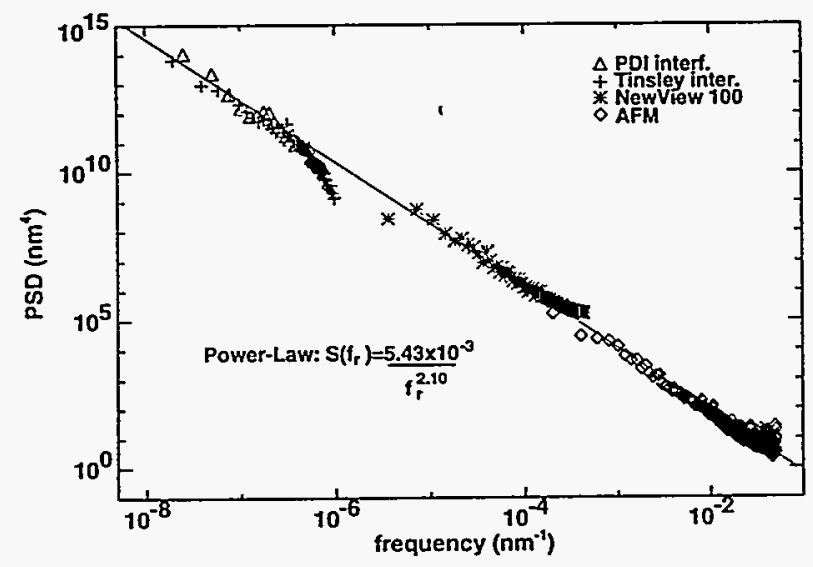

Figure 1. PSD for the concave aspheric substrate M1\#1. control and minimal surface roughness at higher spatial frequencies. Power-law fits to the PSDs are also shown.

Fig. 3. shows PSDs derived from NewView 100 and AFM measurements of the M1\#1 asphere and a spherical optic fabricated for the LLNL EUV lithography station. Data derived from AFM measurements on the highly polished sphere and optical flat are also shown. Using power law fits, [8]

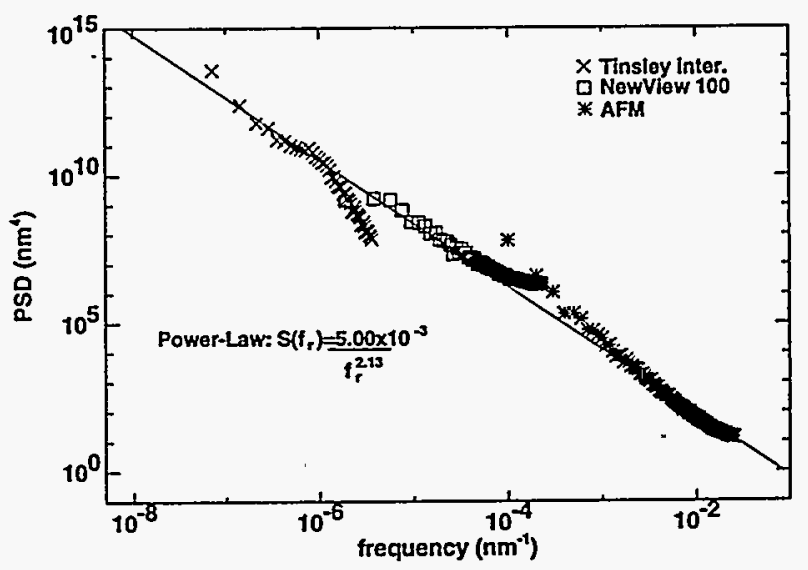

Figure 2. PSD for the convex aspheric substrate M2\#2.

the rms roughness over the mid and high-spatial frequency band, extending from $1.0 \times 10^{-6} \mathrm{~nm}^{-1}$ to $7.7 \times 10^{-2} \mathrm{~nm}^{-1}$ was calculated. The band-limited $\mathrm{ms}$ roughness is $0.062 \mathrm{~nm} \mathrm{~ms}$ for the flat, $0.088 \mathrm{~nm}$ rms for the sphere, $0.64 \mathrm{~nm}$ rms for the LLNL EUVL sphere, and $0.95 \mathrm{~nm}$ for the M1\#1 asphere.

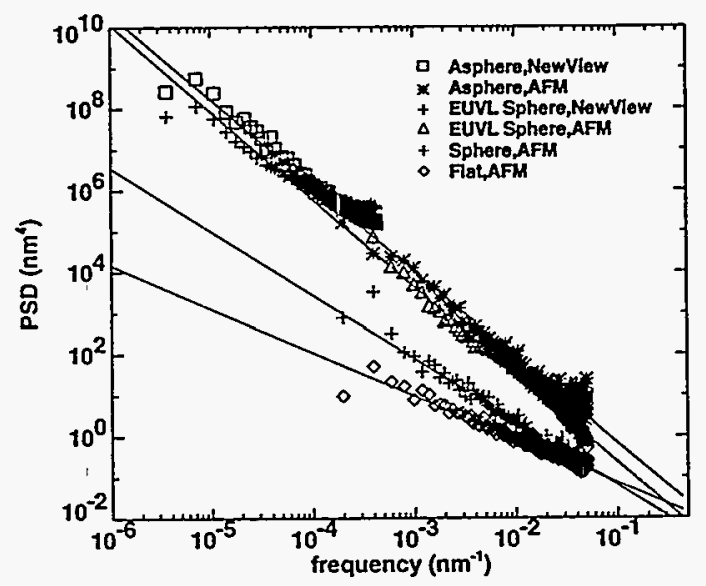

Figure 3. PSD functions at mid and high-spatial frequencies for aspheric (M1\#1) and spherical EUVL imaging mirrors and a sphere and flat with state-of-the-art surface finish. 
The distribution of roughness power in the mid and high-frequency bands is different for the EUVL optics and the highly polished sphere and flat. The distribution of roughness power determines the angular distribution of scattered light in the optical system. If most of the power is concentrated at the higher frequencies, which is the case for the highly polished optics, the scattered light will not reach the image plane.[9] Apart from scattering light out of the

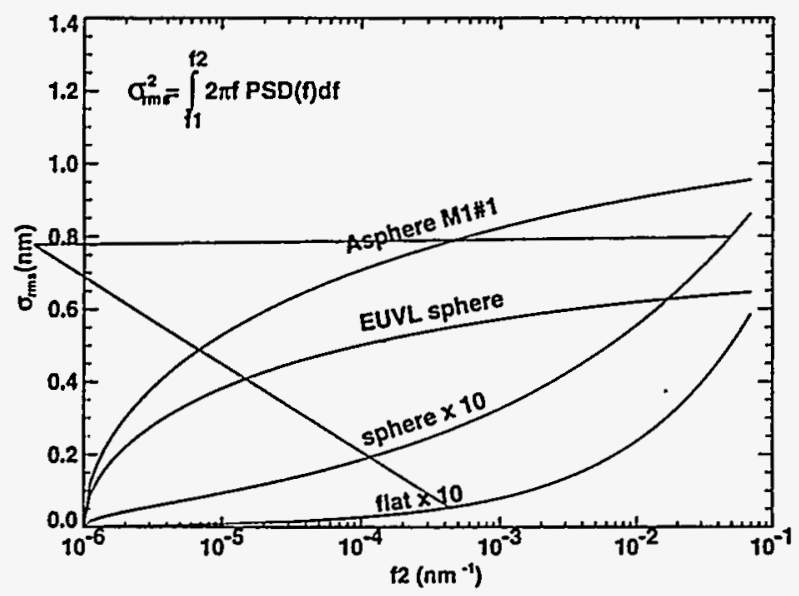

specular beam, these frequencies do not contribute to contrast loss at the image plane. The mid-frequency roughness does scatter light into the image plane, and causes a corresponding loss in contrast. For this reason, mid-spatial frequency roughness is a serious concern for EUV lithography.

The combined mid and high-frequency roughness was computed by integrating power-law fits to the PSD over the varying bandwidths of integration shown in Fig. 4. The lower limit of integration was $1.0 \times 10^{-6}$ $\mathrm{nm}^{-1}$; the upper integration limit is the abscissa. The roughness values for the highly polished sphere and flat have been multiplied by $\times 10$. For example, the sphere would have an rms roughness of $0.088 \mathrm{~nm}$ at $\mathrm{f} 2$ of $7.7 \times 10^{-2} \mathrm{~nm}^{-1}$.

These data show that the amount of roughness power in the EUVL optics is concentrated at the midfrequency range, because the roughness increases rapidly from the lower integration limit and assumes a slower growth rate at higher frequencies. This type of roughness would seriously compromise the achievable. image contrast.
The highly polished substrates have almost no roughness power at the mid-frequencies. The roughness power doesn't increase appreciably until higher frequencies. The primary effect of this type of roughness would be a loss in the radiation transport efficiency of the system.

\section{Conclusions}

State-of-the-art optics fabricated for EUVL have been characterized in terms of surface roughness. The PSD characteristics of both aspheric and spherical optics are indicative of significant mid-frequency roughness. The rms roughness of the M1\#1 concave asphere is 0.95 $\mathrm{nm} \mathrm{rms}$ in the frequency band $1.0 \times 10^{-6} \mathrm{~nm}^{-1}$ to $7.7 \times 10^{-2}$ $\mathrm{nm}^{-1}$. The spherical optic has $0.64 \mathrm{~nm}$ rms roughness. This roughness would significantly compromise imaging performance in an EUVL system. Optics fabricated using advanced polishing techniques exhibited lower roughness and would scatter negligble amounts of radiation. A representative value of 0.088 $\mathrm{nm}$ rms was obtained for a spherical optic.

\section{References and Notes}

1. D.G. Stearns, "Stochastic model for thin film growth and erosion," Appl. Phys. Let., 62, pp1745-1747 (1993).

2. D.G. Stearns, "X-ray scattering from interfacial roughness in multilayer structures," J.Appl.Phys.,71,pp 4286-4298 (1992).

3. D.L. Windt, W.K. Waskiewicz, J.E. Griffith, "Surface finish requirements for soft $X$-ray mirrors, " Appl. Opt., 33, pp 2025-2031 (1994).

4. D.G. Stearns, S. Baker, and M. Wall, "Ultrasmooth, Conducting Multilayer Films for Advanced Applications", presented at The Third International Conference on the Physics of X-ray Multilayer Structures, Breckenridge, Colorado, 37 March, 1996.

5. D.A. Tichenor, et.al.,"Recent results in the development of an integrated EUVL laboratory tool", in Electron-Beam, X-Ray, EUV, and IonBeam Submicrometer Lithographies for Manufacturing V John M. Warlaumont, Editor, Proc. SPIE 2437, pp 292-307 (1995). 
.6. G.E. Sommargren, "Phase Shifting Diffraction Interferometry for Measuring Extreme Ultraviolet Optics," these proceedings.

7. R. Kestner, "Precision Asphere Fabrication and Metrology to Tolerances < $1 \mathrm{~nm}$ RMS", these proceedings.

8. The power law fit for the M1\#1 asphere was based on interferometery, NewView 100, and AFM data. The fit for the LLNL EUVL sphere was based on NewView 100 and AFM data. The sphere and flat with highly polished surfaces were fit using AFM data only.

9. The band of spatial frequencies which scatter light that does not propogate to the image plane, or high-spatial frequencies, is system dependent. Nevertheless, one can estimate the lower cutoff frequency for this band by noting that EUVL systems typically have image-side Numerical Apertures (NA) of 0.1 , which, for a $4 \mathrm{X}$ reduction system, is 0.025 object side NA. Therefore, for 13 $\mathrm{nm}$ radiation, the cutoff frequency is approximately $2 \times 10^{-3} \mathrm{~nm}^{-1}$.

This work was performed under the auspices of U.S Department of Energy by Lawrence Livermore National Laboratory under contract No. W-7405Eng-48. 